\title{
The tumor strikes back: New data on expression of the CD95(APO-1/Fas) receptor/ligand system may cause paradigm changes in our view on drug treatment and tumor immunology
}

\section{Peter H. Krammer}

Tumorimmunology Program German Cancer Research Center, Heidelberg/FRG

In the US, cancer is the second leading cause of death. However, while a decade ago only 10000 patients per year were cured by chemotherapy, at present this rate has increased to 30000 patients per year. Today, there are over 50 chemotherapeutic agents used, singly or combined, in the treatment of malignancy. Efficient drugs include e.g. such diverse chemical compounds as anti-metabolites (e.g. Fluorouracil (5-Fu), methotrexate (MTX)), alkylating agents (mitomycin, cyclophosphamid, cisplatin), intercalators (dactinomycin, mitoxantron, doxorubicin), as well as nucleotide analogues. For years it has been known that these diverse drugs can induce apoptosis. What was not known, however, was the mechanism of apoptosis induced by the drugs. What is more, in the course of drug treatment either primary resistance of the tumor was seen or resistance developed during the course of treatment. The established line of thinking to explain this phenomenon went approximately like this. The chemotherapeutic drugs cause havoc by destroying the genetic material, the DNA of the cell, and impair the cell's ability to replicate and, accordingly, the tumor to grow. Resistance of the tumor against drug treatment and its deleterious consequences for the life of the patients was explained by an efficient DNA repair system, counteracting the drug effects and reconstituting integrity of the genetic material. Furthermore, the cells in a clever anti-drug move had developed pumps, like P-glycoprotein (Pgp) in classic multidrug resistance (MDR) or MDR-associated transporter protein in MRP-associated multidrug resistance, that were thought to busily work against the drugs by pumping 'these poisons' out of the cell. Something, however, did not quite fit with these explanations, mainly the fact that the phenomenon of MDR did not always account for resistance to cancer chemotherapy. In addition, where in all these considerations could one possibly place another player, p53, the 'guardian of the genome', usually but not always upregulated in many but not all drug-treated cells. Two recent papers, published in Nature Medicine by Friesen et al (1996) and in the J. Clin. Invest. by Müller et al (1997), may give a new twist to the story of chemotherapy. These papers show that treatment of leukemic and of hepatocellular carcinoma cell lines, respectively, in vitro with various chemotherapeutic agents causes a dramatic upregulation of the CD95(APO-1/Fas) death system, Figure 1 (Krammer, 1996; Debatin, 1996; Peter et al, 1995). Müller et al (1997) show in addition that upregulation of the CD95 system - following anti-cancer therapy - is preceded and directed by upregulated p53 (in hepatocellular carcino- mas). These data bring together several previous seemingly loose ends in the field of chemotherapy: DNA damage by the chemotherapeutic drugs, the p53 system, the CD95 death system consisting of the CD95 receptor and its ligand, an only partially elucidated signaling cascade (Peter et al, 1996; Schulze-Osthoff et al, 1996; Skowronski et al, 1996) and apoptosis. It is conceivable and, in fact preliminary experiments from the two laboratories would indicate that, as more tumor types will be checked, other death systems such as DR3, TNF-R 1/2, TRAIL, etc. may come into play. But the basic scenario and its impact for tumor treatment will be the same and will cause a change in paradigm in drug treatment and in the evaluation of drug sensitivity and resistance.

The basic scenario is as such: drug treatment causes damage of the DNA in the treated cells, sensed by p53 (or other substitutes in p53 deficient cells) which is upregulated as a consequence. Upregulated p53 then activates directly the promoter for the CD95 receptor and directly or indirectly the promoter of the CD95 ligand or stimulates other death systems. Alternatively, p53 might activate transcription factors which regulate the CD95 receptor gene. Binding of the expressed death ligand to the death receptor will ensue in autocrine apoptotic suicide or in fratricide of

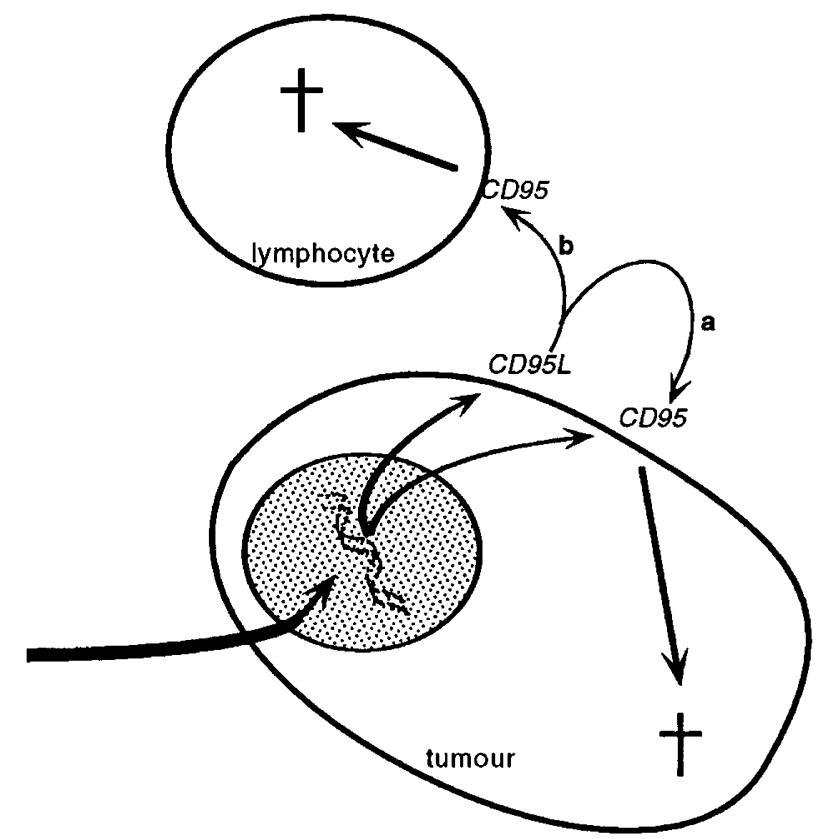

Figure 1 Various chemotherapeutic agents cause an upregulation of CD95 and CD95L, activating both the autocrine (a) and paracrine/fraticide (b) death system. 
neighboring cells. In a successful anti-tumor therapy the CD95 signaling cascade including caspases (SchulzeOsthoff et al, 1996) is switched towards sensitivity. Considerations of drug resistance in the future, therefore, will also have to incorporate testing the functionality of the CD95 and possibly other death systems. It is clear that this has far reaching therapeutic consequences. So far for the good news.

Now comes the bad news. At least one has to contemplate that the above decisive advances in the explanation of the mechanism of chemotherapy may provide an explanation of why chemotherapy could be a double-edged sword, Figure 2. On the one hand, when successful, it helps to kill the tumor. On the other hand, it
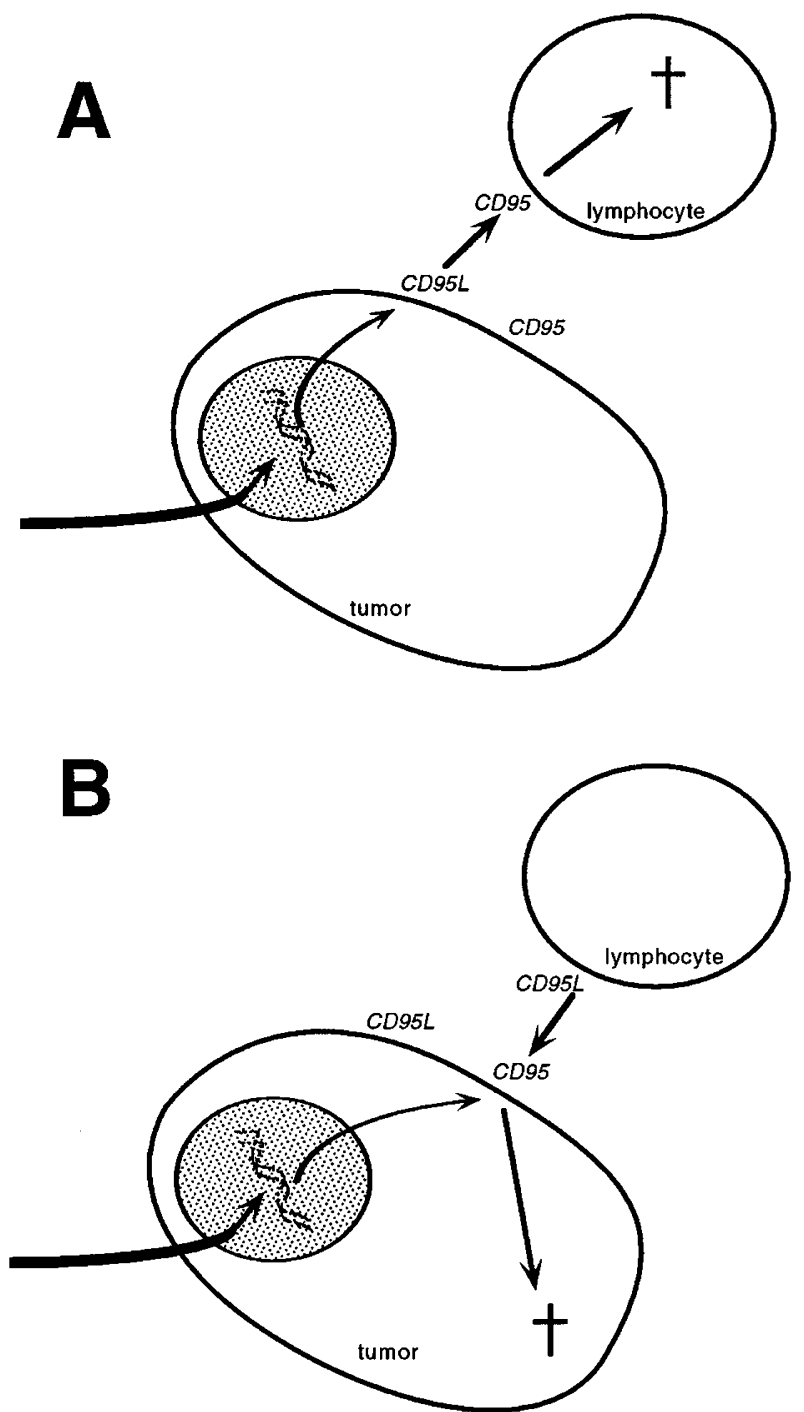

Figure 2 The balance between anti-tumor immunity and tumor counterattack regulates tumor growth. Several molecular mechanisms may be involved in regulating tumor growth including the CD95/CD95L circuit Upregulation of CD95L may provide the tumor with a lethal weapon $(\mathbf{A})$ that kills anti-tumor lymphocytes, and thus boosts immune evasion. This mechanism makes the tumor an immune privileged site. Expression of CD95 helps to kill the tumor by making it more susceptible to lymphocyte killing (B). may provide the tumor with a lethal weapon, boost immune evasion and make the tumor an immune privileged site. The lethal weapon of interest here is the CD95 ligand. The CD95 ligand can be made by tumors constitutively or its expression might be induced, e.g. as detailed above, by drug treatment of the tumor. The ligand might have a deleterious effect on the immune system, particularly on the activated attacker anti-tumor-T-lymphocytes, provided they are in a CD95 apoptosis sensitive state. The experimental background of this situation and its various permutations are outlined in different in vitro and in vivo model systems in four recent papers which, again, may induce a paradigm change, how we look at tumor/host interaction (O'Connell et al, 1996; Hahne et al, 1996; Strand et al, 1996; Seino et al, 1997).

This reasoning provides us basically with a bilateral situation in which (1) attacker T lymphocytes, e.g. activated $\mathrm{T}$ (killer) cells, express two killing systems: the perforin system as well as the CD95(APO-1/Fas) death receptor and ligand, and (2) tumor cells, likewise, express the CD95 ligand and might even have become resistant against its killing activity (e.g. by downregulating CD95) like in most hepatomas (Müller et al, 1997). Previously, the prevailing view was that the $T$ cells recognize the tumor, but do not receive sufficient costimulatory signals and are not properly activated. The new view now adds a different turn. It says that the tumor is not passive but has the weapon to fight the immune system, 'strike back', and cause depletion of attacker lymphocytes and immune suppression. Thus, both fighters, the T cells and the tumor cells, are equipped with a set of almost equal weapons and several parameters may determine who wins the war between them. Apart from the size of the army (number of cells), the quantity of CD95 receptors and ligands and the sensitivity and resistance of CD95 signaling may be important. The best case scenario for the patient is a large pool of $T$ cells equipped with an anti-tumor $\mathrm{T}$ cell receptor repertoire and both killing systems ready to attack a small apoptosis sensitive tumor. The worst case scenario pictures the tumor as the winner. It has become apoptosis resistant, e.g. by downregulating CD95 or by switching the signaling pathway to insensitive, expresses CD95 ligand and meets activated T cells in a CD95 apoptosis sensitive state. The CD95 system stands here for any other system which also might take part in the tumor anti-immune system war. In any case, the net result of such a case could be $T$ cell depletion, immunosuppression, and immune escape. In the future, to turn this situation around to the advantage of the patient and to cause tumor rejection would require means to temporarily induce $\mathrm{T}$ cell resistance towards apoptosis and to maintain sensitivity in the tumor. This will require ingenious experiments and new imaginative approaches.

\section{References}

Debatin K-M (1996) Disturbances of the CD95 (APO-1/Fas) system in disorders of lymphohaematopoietic cells. Cell Death Differ. 3: 185-189

Friesen C, Herr I, Krammer PH and Debatin K-M (1996) Involvement of the CD95(APO-1/Fas) receptor/ligand system in drug induced apoptosis in leukemia cells. Nature Medicine 2: $574-577$ 
Hahne M, Rimoldi D, Schröter M, Romero P, Schreier M, French LE, Schneider P, Bornand T, Fonatan A, Lienard D, Cerottini J-C and Tschopp J (1996) Melanoma cell expression of Fas(APO-1/CD95) ligand: implications for tumor immune escape. Science 274: 1363

Krammer PH (1996) The CD95(APO-1/Fas) receptor/ligand system: death signals and diseases. Cell Death Differ. 3: 159-160

Müller M, Strand S, Hug H, Heinemann E-M, Walczak H, Hofmann WJ, Stremmel W, Krammer PH and Galle PR (1977) Drug-induced apoptosis in hepatoma cells involves activation of $p 53$ and is mediated by the CD95/(APO-1/Fas) receptor/ ligand system. J. Clin. Invest. 99:1-11

O'Connell J, O'Sullivan GC, Collins JK and Shanahan F (1996) The Fas counterattack: Fas-mediated T cell killing by colon cancer cells expressing Fas ligand. J. Exp. Med. 184: 1075-1082

Peter ME, Hellbardt S, Schwartz-Albiez R, Westendorp MG, Walczak $H$, Moldenhauer G, Brell M and Krammer PH (1995) Cell surface sialylation plays a role in modulating sensitivity towards APO-1-mediated apoptotic cell death. Cell Death Differ. 2: 163-171
Peter ME, Kischkel FC, Hellbardt S, Chinnaiyan AM, Krammer PH and Dixit VM (1996) CD95 (APO-1/Fas)-associating signalling proteins. Cell Death Differ. 3 : $161-170$

Schulze-Osthoff K, Bauer MKA, Vogt M and Los M (1996) Role of ICE-realted and other proteases in Fas-mediated apoptosis. Cell Death Differ. 3: 177-184

Seino K-I, Kayagaki N, Okumura K and Yagita H (1997) Antitumor effect of locally produced CD95 ligand. Nature Med. 3: 165-170

Skowronski EW, Kolesnick RN and Green DR (1996) Fas-mediated apoptosis and sphingomyelinase signal transduction: the role of ceramide as a second messenger for apoptosis. Cell Death Differ. 3: 171-176

Strand S, Hofmann WJ, Hug H, Müller M, Otto G, Strand D, Mariani SM, Stremmel W, Krammer PH and Galle PR (1996) Lymphocyte apoptosis induced by CD95 (APO-1/Fas) ligand expressing tumor cells - a mechanism of immune evasion? Nature Med. 12: 1361-66 